\title{
Carnets
}

Revue électronique d'études françaises de l'APEF

Deuxième série - 8 | 2016

Du Français en cause aux causes du français

\section{Pour un enseignement/ apprentissage contextualisé de la "grammaire » du français langue étrangère}

Henri Besse

\section{OpenEdition}

Journals

Édition électronique

URL : http://journals.openedition.org/carnets/1858

DOI : $10.4000 /$ carnets. 1858

ISSN : 1646-7698

Éditeur

APEF

Référence électronique

Henri Besse, «Pour un enseignement/ apprentissage contextualisé de la « grammaire » du français langue étrangère ", Carnets [En ligne], Deuxième série - 8 | 2016, mis en ligne le 30 novembre 2016, consulté le 30 avril 2019. URL : http://journals.openedition.org/carnets/1858 ; DOI : 10.4000/ carnets. 1858

Ce document a été généré automatiquement le 30 avril 2019.

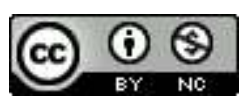

Carnets est mis à disposition selon les termes de la licence Creative Commons - Atribution - Pas d'utilisation commerciale 4.0 International. 


\title{
Pour un enseignement/ apprentissage contextualisé de la " grammaire » du français langue étrangère
}

\author{
Henri Besse
}

1 Après avoir quelque peu précisé les termes de cet intitulé (en 1), nous traiterons de la polysémie actuelle du mot grammaire dans les langues européennes (en 2), des conceptions qu'on se fait de ce qu'est une règle grammaticale (en 3), et des manières de les enseigner ou de les faire apprendre (en 4). Quatre parties, suivies d'une brève conclusion portant sur ce que nous appelons la « culture grammaticale » du français.

\section{Quelques précisions quant à l'intitulé de cet atelier}

2 Pourquoi cette barre entre enseignement et apprentissage, enseigner ne signifie-t-il pas «faire apprendre»? Pourquoi contextualisé, tout enseignement comme tout apprentissage n'est-il pas inévitablement « contextualisé »? Pourquoi ces guillemets au mot «grammaire»? Pourquoi s'en tenir au français, alors qu'apprendre scolairement une langue étrangère ou seconde (L2), autre que celle(s) qu'on pratique déjà, sa langue maternelle (L1) et éventuellement une première L2, engage, tout aussi inévitablement, plusieurs langues?

\section{Pourquoi enseignement / apprentissage ?}

3 La barre entre enseignement et apprentissage signifie qu'il s'agit de deux processus distincts qui n'ont d'existence, en classe de L2, qu'au sein de multiples interactions, plus ou moins individualisées, entre l'enseignant et le groupe-classe mais aussi entre les enseignés. Interactions dont l'affectif et l'émotif ne sont pas absents, qui font que le dûment enseigné est ou non appris, qu'il l'est de telle façon par tel élève et d'une autre façon par 
tel autre. Façons d'apprendre parfois très éloignées de l'idée que l'enseignant peut s'en faire. Dans une classe de L2, peut-être davantage que pour d'autres matières scolaires, les élèves apprennent à la fois plus et moins que ce qui leur est enseigné.

On ne peut donc concevoir cet enseignement comme la simple transmission par le maître d'un savoir d'origine savante à ceux qui l'ignorent. Et ce savoir ne peut être inculqué selon sa seule « logique » interne, sans que le maître n'ait à s'interroger sur les manières dont ses élèves sont à même ou non de se l'approprier. Il est clair que cette conception de l'enseignement / apprentissage requiert des classes à effectif relativement réduit, et convient mieux à l'enseignement de certaines matières (dont les L2) qu'à d'autres.

\section{Pourquoi un enseignement / apprentissage contextualisé ?}

Par contextualisé, il faut entendre ici un enseignant attentif à ce qu'engage, pour chacun de ses élèves et dans le ici et maintenant de leur classe, l'apprentissage de ce qu'il cherche à leur enseigner. En d'autres termes, un enseignant qui s'efforce de prendre en compte, selon les réactions de ses élèves et autant qu'il lui est possible, ce qui lui paraît être à même de leur permettre de s'approprier, avec plus ou moins de succès, les savoirs pratiques ou plus savants qu'il cherche à leur faire apprendre. Dans cet article, il s'agit d'un savoir sinon savant du moins d'origine savante, celui des grammairiens ou des linguistes dont le maître sait, au moins par l'expérience qu'il en a, qu'il ne permet pas, à lui seul, de pratiquer une L2 à l'égal des natifs de celle-ci, dont certains ignorent tout de ce dit savoir. Ce savoir apparaît néanmoins nécessaire - pour certains élèves plus que d'autres, pour certaines L2 plus que d'autres - à l'appropriation réussie d'un parler-écrire étranger.

6 En outre, il est illusoire de penser qu'on puisse enseigner / apprendre une L2, sans prendre compte, d'une manière ou d'une autre, les langues (dont leur L1) que les élèves pratiquent déjà, et à partir desquelles ils apprennent, même si le maître ne les utilise pas en classe et s'il leur interdit de les parler ouvertement durant ses cours ${ }^{1}$. Passé la prime enfance, il semble ${ }^{2}$ en effet qu'on reste apte, génétiquement, à apprendre une L2 parce qu'on pratique déjà au moins une autre langue (sa L1). L'apprentissage d'une L2 engage donc inévitablement, passée la prime enfance, au moins la L1 des élèves, et parfois une autre L2 qui leur est déjà plus ou moins familière. Et s'ils les ont aussi apprises scolairement, cet apprentissage engage souvent des rudiments du savoir des grammairiens ou des linguistes sur ces langues qui leur permettent d'apprendre une nouvelle (pour eux) L2.

D'où de multiples interférences linguistiques que le maître s'efforce, avec plus ou moins de réussite, de corriger, mais aussi des interférences d'ordre métalinguistique, qui sont liées aux « règles " grammaticales qu'ils ont déjà apprises à propos de leur L1 ou de leur première L2. "Règles " qui engagent des catégories et raisonnements grammaticaux qu'ils ont tendance à re-mobiliser pour surmonter leurs difficultés d'appropriation de cette nouvelle L2, et qui interfèrent parfois avec les «règles" qu'on leur a déjà enseignées. Ce qui ne manque pas d'entraver leur appropriation de cette L2. C'est aussi en ce sens que nous parlons, dans cet article, d'enseignement / apprentissage contextualisé. On ne peut, à notre avis, enseigner la "grammaire» d'une L2 sans tenir compte non seulement des régularités et analogies propres à la L1 (et éventuellement de celles d'une première L2) des élèves, mais aussi de ce qu'ils ont appris de " grammatical », au sens plus savant du terme, sur leur L1 (éventuellement sur leur première L2). Dit plus simplement, 
on ne peut enseigner le savoir que les grammairiens ou les linguistes ont élaboré à propos de la L2 qu'on leur enseigne, sans tenir compte du « passé grammatical » de ses élèves, de ce qu'ils ont retenu du savoir d'autres grammairiens et linguistes qui leur a été déjà scolairement enseigné à propos de leur L1 ou d'une première L2.

\section{Pourquoi des guillemets à « grammaire » ?}

8 Une classe de L2 n'est donc pas une classe où l'enseignant se borne à transmettre à ses élèves le savoir grammatical ou linguistique que les spécialistes ont élaboré à propos de cette seule langue, la grammaire des livres et des cours qui l'ont académiquement qualifié en tant que « maître » pour enseigner cette L2. Il nous semble d'ailleurs que des maîtres qualifiés pour enseigner deux langues (leur L1 et une L2, ou deux L2) sont mieux à même d'affronter la complexité de l'apprentissage de la L2 qu'ils enseignent que les natifs de cette langue ou les maîtres académiquement qualifiés pour enseigner une seule L2. Si ce savoir d'origine savante sur la L2 peut aider les élèves à s'approprier correctement cette L2, il ne permet pas à lui seul, d'évidence, de la pratiquer avec l'aisance, en particulier dans ses composantes phonétiques et grammaticales, du petit enfant qui parle sa L1, dont on dit parfois de nos jours qu'il pratique déjà la grammaire de sa langue maternelle ${ }^{3}$.

Le mot grammaire peut ainsi renvoyer - et il en va à peu près de même de ses équivalents dans les autres langues européennes - à au moins trois réalités différentes. 1) Aux régularités internes propres à une langue (on parle ainsi de la grammaire du français, de l'anglais, $d u$ portugais...), aux caractéristiques d'ordre morpho-syntaxique qui la distinguent des autres langues. 2) À la compétence ou à l'intuition de qui pratique ces régularités sans les avoir dûment étudiées, c'est ainsi qu'il faut entendre grammaire si l'on dit (voir ci-dessus) que l'enfant, avant d'aller à l'école, possède la grammaire de sa langue maternelle. 3) Aux représentations explicitées que donnent les spécialistes, grammairiens ou linguistes, d'une partie ${ }^{4}$ de ces régularités (un livre de grammaire, un article de grammaire ...). Ou encore 4), aux points de vue qu'adoptent ces spécialistes pour étudier méthodiquement ces régularités (on parle ainsi de grammaire traditionnelle, structurale, fonctionnelle, générative, énonciative...). D'où nos guillemets au mot grammaire, un des objectifs de notre atelier étant de clarifier les rapports qu'entretiennent entre elles ces différentes sortes « grammaires ».

\section{Pourquoi n'est-il question que du français?}

10 S'il n'est question, dans notre titre, que du français L2, c'est d'abord que cet atelier s'adressait à des enseignants du français L2 et qu'il s'est déroulé en français. Mais c'est aussi parce que chaque langue entretient dans ses pratiques, tant usuelles que d'enseignement, un rapport particulier au savoir que les grammairiens et les linguistes ont développé à son propos. Rapport d'ordre moins scientifique que culturel, en ce qu'il est moins lié à l'histoire interne d'une langue qu'à son histoire externe, au rôle que ce savoir a joué et continue à jouer dans les usages plus ou moins ordinaires de cette langue, et donc dans la société qui s'identifie et est identifiée à celle-ci.

11 Il est, en effet, des langues où ce savoir d'origine savante a été plus fortement intégré aux pratiques quotidiennes de ceux et celles qui les parlent-écrivent que dans d'autres. Où ce savoir a été plus largement vulgarisé que pour d'autres, où sa naturalisation ou intégration dans le parler-écrire de leurs locuteurs, qu'ils en soient natifs ou non, est plus 
manifeste, plus prégnante que dans d'autres. Autrement dit, il est des communautés qui accordent à leur culture grammaticale une place plus importante que d'autres 5 . Au sein des langues européennes, ceux et celles dont le français est la langue maternelle ou seconde sont en général plus attentifs à la culture grammaticale de leur langue que, par exemple, ceux et celles dont l'anglais est la langue maternelle ou seconde. Nous reviendrons (voir Besse 2001) brièvement sur ce point dans notre conclusion.

\section{Retour sur les ambiguïtés du mot grammaire}

Réfléchir à l'enseignement / apprentissage de la " grammaire » d'une L2 suppose que l'on revienne sur la polysémie du mot grammaire. Les dictionnaires de linguistique lui accordent jusqu'à six ou sept sens différents, mais notre sentiment est qu'il suffit de distinguer trois sens fondamentaux pour que cette réflexion en soit clarifiée.

\section{La grammaire en tant que grammaire intériorisée}

13 Le premier est le sens que prend grammaire dans une phrase comme: Toute langue a une grammaire, et l'enfant pratique la grammaire de sa langue maternelle avant même d'aller à l'école. Phrase qui aurait sans doute étonné au XVII et même au XVIII siècle (voir ci-dessus la note 3), car elle donne à grammaire une acception que ce mot n'avait guère jusqu'alors. C'est qu'elle en fait un quasi synonyme de langue ("toute langue a une langue $\left.{ }^{6} »\right)$. Grammaire peut y être paraphrasé par: toute langue a son propre système, son organisation interne spécifique, sa structure, ses propres règles...

Pris en ce sens, le mot postule qu'il existe dans la nature de chaque langue une certaine rationalité, qu'on peut y observer des régularités ou des analogies dans ses énoncés tant oraux qu'écrits, lesquelles sont suivies plus ou moins régulièrement par nombre de ses natifs qui, pourtant, ne les ont pas étudiées rationnellement.

C'est que le grammairien ou le linguiste se donne un objet autre que ces énoncés dont le sens utile ou communicatif est fortement tributaire des situations et des contextes dans lesquels ils sont échangés. Il ne tient pas compte, le plus souvent, de qui en fait usage, à qui ces énoncés sont destinés, dans quel but et dans quel environnement (physique ou pensé) ils ont été produits et reçus. Il les extrait de cet environnement pour en faire des propositions ou des phrases, qu'il étudie pour y trouver des ressemblances, d'ordre formel et/ou sémantique, sans trop se préoccuper de ce qu'elles peuvent effectivement signifier dans les échanges ordinaires de ses natifs. Pour la plupart des grammairiens et des linguistes, j'ai chaud signifie littéralement « avoir chaud » et non " ouvre la fenêtre » ou " excusez-moi d'enlever ma veste »; j'ai chaud peut alors être rapproché, entre autres, de j'ai froid, faim, peur, mais aussi tu as chaud, froid, peur, il a chaud, froid, peur. Ce qui permet à ces grammairiens et linguistes de regrouper ces propositions ou phrases selon ce qu'ils appellent des paradigmes dont on peut se demander s'ils relèvent de la grammaire intériorisée de ceux qui pratiquent cette langue sans les connaître ou sans en avoir conscience, ou bien s'ils ne relèvent pas d'une « logique " propre à ces doctes. Ce n'est donc pas la réalité langagière dans sa diversité et complexité communicative qu'étudie le grammairien ou le linguiste, mais une abstraction de cette réalité qui en fait un objet devenu rationnellement observable d'un point de vue qui n'est pas nécessairement celui de tous les locuteurs de la langue qu'il étudie. Ce que nous qualifions de grammaire intériorisée d'une langue n'est pas cet objet, pour ainsi dire inerte, que se donne le 
grammairien ou le linguiste afin d'en dégager des règles. C'est ce qui résulte dans l'esprit d'un locuteur de cette langue de l'intériorisation, plus ou moins singulière, qu'il a faite, au travers de multiples interactions avec ceux (celles) qui les maîtrisent déjà, des usages ordinaires de cette langue. Grammaire intériorisée qui lui permet d'affirmer, au moins intuitivement, que j'ai chaud se dit en français, alors que *j'ai vert ou *j'ai voiture ne se disent pas.

Il y a de l'inné et de l'acquis dans une " grammaire intériorisée ». L'inné est ce qui permet à tout être humain, pour peu que son environnement quotidien l'y aide, de parler une langue (L1 ou L2) comme on la parle. Cet inné relève de la faculté de langage comme disaient jadis les philosophes; du Language Acquisition Device ("dispositif inné d'acquisition des langues ») comme disent certains linguistes actuels. L'acquis est ce qui résulte dans l'esprit d'un enfant ou d'un adulte, grâce à cet inné, de l'intériorisation progressive de ce qui est plus ou moins propre à une langue, que cette intériorisation relève, à l'oral, de sa phonétique, de ses intonations, de sa gestualité, et à l'écrit, de son écriture et sa lecture. En d'autres termes, cet inné relève de l'universel humain, de sa génétique, et cet acquis de ce qui divise l'espèce humaine en quelque six mille langues différentes, non ou peu inter-compréhensibles ${ }^{7}$.

Par grammaire intériorisée, nous entendons donc que tout être humain dispose génétiquement de la capacité d'acquérir, aisément dans sa prime enfance et moins aisément plus tard, n'importe quelle langue humaine, pour peu qu'il soit contraint, dans l'environnement où il vit, d'interagir avec ceux et celles qui la parlent-écrivent quotidiennement. Ce sont ces multiples interactions qui lui permettent, peu à peu et non sans approximations ou généralisations abusives ${ }^{8}$, de s'approprier, sans qu'elles lui soient enseignées d'un point de vue grammatical, les régularités ou analogies caractéristiques de cette langue qui lui était jusqu'alors étrangère. Dans une classe de L2, c'est d'abord parce que l'enseignant a déjà intériorisé, nativement ou non, avec ou non le secours du savoir des grammairiens ou des linguistes, les régularités et analogies propres à cette L2 qu'il est à même d'aider les apprenants à les intérioriser?.

\section{La grammaire en tant que représentations grammaticales plus ou moins savantes}

Le second sens du mot grammaire que nous retenons est celui qu'il prend dans un manuel de grammaire française ou portugaise, c'est-à-dire dans un ouvrage qui vise à vulgariser ce que les grammairiens ou les linguistes ont dit de "grammatical» sur cette langue, en particulier les règles qu'ils ont élaborées à son propos. C'est aussi le sens que peut prendre ce mot dans Ce sont des langues qui n'ont pas encore de grammaire, si l'on entend par là que ces langues, n'ayant pas été étudiées méthodiquement par les grammairiens ou les linguistes, ne disposent pas encore de règles grammaticales, demeurant régies par leurs seuls usages ${ }^{10}$. Pour qualifier ce sens, nous parlons de représentations grammaticales plus ou moins savantes.

Représentation, en raison du fait que les règles explicitées dans les manuels de ce type ne sont pas, à notre avis, une simple description des régularités ou des analogies dont le grammairien ou le linguiste cherche à rendre compte. Pas plus qu'elles ne sont des « lois » jusqu'alors cachées que ces spécialistes auraient enfin redécouvertes. Et comme il n'est pas rare que, pour un même phénomène observable dans les usages attestés d'une langue, 
on dispose de différentes règles selon les grammairiens ou les linguistes qui les ont forgées, nous parlons de ces représentations au pluriel ${ }^{11}$.

Ces règles sont formulées dans des termes métalinguistiques qui renvoient à des notions abstraites, qui sont parfois empruntées aux philosophes et parfois inventées par tel ou tel grammairien ou linguiste, termes qu'il est nécessaire, en classe de L1 comme en classe de L2, d'expliquer aux élèves. Une manière courante d'y parvenir, tant dans les manuels que dans les classes, est de recourir à des exemples à même de montrer à quel réel observable s'applique la règle enseignée, ces exemples étant supposés prouver la règle. Si un élève avance un contre-exemple, on lui rétorque que "l'exception prouve la règle", oubliant que prouver vient du latin probare, lequel signifiait d'abord "mettre à l'épreuve", " éprouver ». Notons enfin que, dans une classe de L2, ces exemples sont souvent moins éclairants que dans une classe de L1, pour la simple raison que les élèves ont de la difficulté à les comprendre, ou qu'ils les comprennent autrement que les grammairiens ou les linguistes. Ce n'est donc pas le savoir des grammairiens et des linguistes tel qu'ils l'ont eux-mêmes formulé qui est présent dans une classe (de L1, et encore moins dans une classe de L2), mais un savoir qui y est transposé à des fins didactiques. C'est pourquoi nous parlons de représentations grammaticales plus ou moins savantes ou d'origine savante.

\section{La grammaire en tant que théories grammaticales}

21 Le troisième sens que nous retenons est celui que prend le mot grammaire dans, par exemple, Il existe différentes grammaires, fonctionnelle, structurale, générative, énonciative... Il renvoie alors aux "écoles de pensée ", aux points de vue théoriques qu'adoptent les grammairiens ou les linguistes dans leur étude «scientifique» d'une langue, plus rarement de plusieurs.

Les grammairiens, ceux qui œuvrent sur une seule langue et en élaborent une représentation relativement exhaustive de ses composantes " grammaticales ${ }^{12}$, sont, en général, discrets sur leurs assomptions ou présupposés théoriques. Ils ont tendance à présenter leurs règles comme si elles existaient dans la réalité même de la langue, écrivant plus volontiers: "il existe en français trois espèces d'articles ", que "on peut distinguer en français... » ou " notre hypothèse est qu'il y a en français... ». Une des raisons de cette discrétion théorique est qu'ils s'inscrivent souvent dans une tradition grammaticale qui a fini, au cours des siècles, par s'imposer dans l'étude de la langue qu'ils ont prise pour élaborer leur objet d'étude, tradition qu'ils ne remettent pas radicalement en cause, se bornant à quelques innovations. Les linguistes, qui prétendent à plus de généralité et qui travaillent parfois sur plusieurs langues, sont davantage diserts quant à ce qui est préalable à leur travail.

23 Au point que certains linguistes consacrent l'essentiel de leur dire savant à la défense et illustration de la théorie dont ils sont adeptes : plus rarement, de celle qu'ils ont euxmêmes élaborée. À quoi s'ajoute que les linguistes ne cherchent pas à élaborer une représentation de l'ensemble des phénomènes grammaticaux d'une langue, mais s'en tiennent à des aperçus portant sur quelques points à même d'étayer leur théorie ou d'en montrer l'applicabilité. Ils ne sont pas, à quelques exceptions près, auteurs de manuels de grammaire mais d'ouvrages de linguistique. Lesquels ne sont pas, en tant que tels, utilisables dans l'enseignement / apprentissage d'une langue, Et nombre d'entre eux ont tendance à présenter la théorie dont ils se réclament, tant dans leurs écrits que dans leurs cours universitaires, comme une sorte de "vérité » des langues dont cette théorie serait 
l'explicitation. Prétention qui a été fortement contestée dans d'autres domaines ${ }^{13}$, et qui nous paraît tout aussi contestable dans l'étude, fût-elle réputée scientifique, des langues.

Mais si une théorie linguistique n'est pas à même de dire "la vérité » des langues, elle n'en est pas moins indispensable à qui cherche à les étudier rationnellement. Elle précise le point de vue que le linguiste ou le grammairien adopte quant à la manière de recueillir et d'étudier les données sur lesquelles il travaille, et elle permet de définir les catégories et les raisonnements qu'il utilise dans son étude. Elle constitue donc l'outillage conceptuel indispensable à son travail. Et si l'on a parfois l'impression que certains se passent de ces préalables spéculatifs et de ces outils abstraits, c'est qu'ils s'inscrivent dans une tradition théorique qu'ils présument déjà familière à ceux à qui ils s'adressent, ou bien qu'ils présupposent inhérente à l'esprit humain, ou encore qu'elle émane, par une induction généralisante, de la langue observée. Ce qui aboutit parfois, à ce que ces linguistes font comme si l'on ne pouvait, en matière de "grammaire ", penser autrement qu'eux.

Ce qui, d'évidence, n'est pas la meilleure façon de comprendre ce qui peut se passer dans une classe de L2. Certains élèves peuvent en effet être accoutumés à une autre tradition grammaticale que celle de leur manuel ou de leur maître ${ }^{14}$. D'autres relèvent de cultures qui ignorent traditionnellement le savoir des grammairiens ou des linguistes, ce qui fait qu'ils n'y ont pas été accoutumés dans l'étude scolaire de leur L1 ${ }^{15}$. Et d'autres encore viennent de systèmes scolaires où, dans le primaire et le secondaire, il n'y a pas ou il n'y a que très peu de cours de grammaire, le savoir des grammairiens et des linguistes relevant d'études universitaires spécialisées ${ }^{16}$. Enfin, même quand des élèves ont été formés dans une tradition grammaticale proche de celle des manuels de français L2, il arrive souvent que la proximité apparente des terminologies soit moins une aide qu'un piège, que ces termes savants soient d'aussi « faux-amis » que nombre de mots courants. Bref, enseigner comme allant de soi un savoir qui représente scientifiquement les usages d'une langue en tant que L1, c'est ne pas tenir compte de la réelle étrangeté pour certains élèves de ce savoir, et ce n'est pas, d'évidence, la meilleure façon de leur en faciliter l'apprentissage.

\section{Qu'entend-t-on par règles grammaticales d'une langue?}

Toute réflexion didactique visant à aider les élèves à intérioriser les régularités ou analogies caractéristiques d'une L2 implique de s'interroger sur les relations qu'entretiennent les trois sortes de savoirs grammaticaux que nous venons de distinguer : le savoir pratique ou intuitif lié à la grammaire intériorisée d'une langue ; le savoir, plus savant ou déclaratif, des représentations grammaticales que les grammairiens ou les linguistes donnent de certaines composantes de cette langue; le savoir grammatical théorique, d'ordre plus spéculatif, à partir desquels ces mêmes spécialistes ont élaboré leur savoir. De manière empirique, on peut distinguer trois façons de comprendre ou de raisonner les relations entre ces trois types de savoirs grammaticaux.

À partir de notre propre expérience d'apprentissage des L2, des réactions et des remarques des (futurs) enseignants de français L2 que nous avons eus en formation, de ce que les grammairiens et les linguistes ont pu dire des règles qu'ils élaborent et de ce qu'en ont écrit quelques écrivains, nous avons élaboré un questionnaire comprenant six « définitions » de ce que peut être une règle de grammaire. À chacune de nos trois façons 
de concevoir ce qu'est une règle, correspondent, dans ce questionnaire, deux "définitions" plus ou moins paraphrastiques (voir ci-après). Nous utilisons ce questionnaire à des fins heuristiques, pour aider les (futurs) enseignants de français L2 à prendre conscience, en cochant les «définitions» qui correspondent le mieux à leur conception ce qu'est une règle de grammaire. Et partant, à prendre conscience du type de relations qu'ils établissent mentalement entre ce que nous appelons grammaire intériorisée, représentations grammaticales plus ou moins savantes et théories grammaticales. Et comme ils cochent souvent des «définitions » correspondant à deux, rarement plus, de nos trois conceptions, et qu'ils peuvent parfois interpréter nos « définitions » autrement que nous, nous parlerons de leur (pré)conception des règles grammaticales.

\section{Une (pré)conception d'ordre juridique}

28 Elle revient à concevoir les règles grammaticales comme équivalant, dans les pratiques usuelles d'une langue, aux « lois » telles qu'on les entend dans les domaines du droit, de la morale ou de la religion. Dans notre questionnaire ${ }^{17}$, les deux "définitions" qui correspondent à cette (pré)conception sont : C'est un précepte pour bien parler et écrire une langue et C'est un énoncé qui préconise comment l'on doit parler/écrire une langue.

C'est à cette (pré)conception que renvoie, nous semble-t-il, Marguerite Yourcenar dans ses Mémoires d'Hadrien: "La grammaire, avec son mélange de règle logique et d'usage arbitraire, propose au jeune esprit un avant-goût de ce que lui offriront plus tard les sciences de la conduite humaine, le droit ou la morale, tous les systèmes où l'homme a codifié son expérience.» Traditionnellement, les grammairiens du français font de la grammaire, "l'art de bien parler et de bien écrire", et c'est encore le premier sens, qualifié de "vieux mais courant", que lui confèrent nos dictionnaires actuels (nous soulignons) : « Ensemble des règles à suivre pour parler et écrire correctement une langue. ${ }^{18}$ $\gg$

Cette (pré)conception de la grammaire est souvent dite normative, en ce qu'elle surimpose aux usages ordinaires d'une langue, usages relativement diversifiés et variables selon les contextes d'emploi, une sorte de code qui en dit le «bon usage » et qui, partant, réduit la variabilité et diversité des usages effectivement attestés, ce qui contribue à les normer ou à les standardiser. Le travail du grammairien s'apparente alors à celui du juriste qui codifie en droit écrit, en lois positives (au sens juridique de ces termes), les us et coutumes antérieurement pratiqués sans code. Claude Fabre de Vaugelas se voulait ainsi le greffier ${ }^{19} \mathrm{du}$ " bon usage ", et $\mathrm{M}$. Grevisse s'est inscrit dans cette tradition. Les règles du grammairien sont alors des prescriptions qui, tout comme les lois du juriste, sont émises par une autorité reconnue dans une société donnée, et qui entrainent sanction, à l'intérieur de cette société, pour qui ne s'y conforme pas. La facture même d'un ouvrage intitulé grammaire (de telle langue) n'est alors guère différente de celle d'un recueil de lois ou d'un code juridique (pour tel pays), l'énoncé de la règle y précédant les exemples qui la justifient et les exceptions qui la limitent, à la manière dont la loi est complétée par la jurisprudence de ceux qui l'ont effectivement appliquée à des cas non prévus par la loi.

31 Cette conception de la règle de grammaire a longtemps été dominante chez les grammairiens des langues européennes, et l'est encore chez certains. Mais dans les groupes d'enseignants de français L2 natifs de cette langue peu nombreux sont ceux qui, dans notre questionnaire, cochent l'une ou l'autre des deux " définitions » renvoyant à cette conception. Leur culture actuelle, en ce domaine comme dans d'autres, semble 
répugner à reconnaître l'utilité, même pédagogique, de la normativité. En revanche, ce sont ces deux "définitions" qui sont majoritairement cochées dans les groupes d'enseignants de français L2 relevant des pays arabophones. Peut-être faut-il voir là un effet de la façon dont les règles de l'arabe non dialectal, «l'arabe classique », leur sont traditionnellement enseignées.

\section{Une (pré) conception descriptiviste} strictement de ce "positivisme scientifique ", mais elle lui doit quelque chose. Elle en une sorte de vulgarisation aux sciences qu'on ne disait pas encore « humaines et sociales ». Vulgarisation qui pose que le savoir « positif » relève, dans ces sciences comme dans celles de la nature, d'une observation présumée indépendante de l'observateur, coupées de sa subjectivité et de ses diverses croyances, qu'elle soient religieuses ou autres. Une observation pour ainsi dire sans observateur, apurée de tout a priori, qui serait seule à même de dire objectivement ce qu'est le réel. D'où notre qualificatif descriptiviste.

Une formule du grammairien Ferdinand Brunot, avancée dans un «cours de méthodologie » donné en Sorbonne en 1909 à destination d'enseignants de français (L1 et L2), résume bien ce qui sous-tend une telle conception: «les lois scientifiques de la linguistique historique ou comparée ne sont que des faits observés et généralisés » (1919: 35). Conception qu'on retrouve, officialisée pour les instituteurs et institutrices de France, dans l'article Grammaire du Dictionnaire de Ferdinand Buisson (1911 : 747) : il existe « des faits de langage qu'on ne peut que constater» parce qu'ils sont inexplicables logiquement; il faut donc, «au point de vue pédagogique », délaisser "la méthode dogmatique » pour adopter «la méthode expérimentale, qui dégage les faits 
grammaticaux de l'étude de textes empruntés à nos bons écrivains et convenablement choisis en vue de la leçon ».

Le groupe d'enseignantes portugaises de français L2 qui a suivi notre atelier en juillet 2016 a très majoritairement coché, fait relativement rare dans les groupes avec lesquels nous avons travaillé, les deux "définitions " correspondant à notre (pré)conception descriptiviste. Ces "définitions" conduisent, en effet, le plus souvent à ce genre de " méthode expérimentale ", à " dégager » ou à " induire » les règles grammaticales de la L2 à partir d'exemples choisis par le maître à cet effet. Démarche que nous disons de grammaire inductive explicitée, l'explicitation de la règle impliquée (du latin implicare, littéralement " envelopper ", " plier dans ») dans les exemples pouvant être faite par le maître, par les élèves, et le plus souvent conjointement par celui-là et ceux-ci.

\section{Une (pré) conception constructiviste}

Dans cette conception, la règle grammaticale est une sorte d'artefact du grammairien ou du linguiste, en ce qu'elle dépendrait autant du réel qu'il observe que du point de vue à partir duquel il l'observe. Point de vue à l'origine de divers outils conceptuels qui lui permettrait non plus de découvrir des règles qui seraient cachées dans la nature des langues, mais d'en inventer qui permettraient de modéliser mentalement leurs apparentes régularités afin, entre autres, de pouvoir les informatiser. Les deux « définitions » qui, dans notre questionnaire, lui correspondent sont: C'est une hypothèse, formulée par le grammairien ou le linguiste, quant à la façon dont les mots d'une langue se combinent en proposition ou en phrase; C'est une formulation qui explicite la façon dont on suppose que fonctionnent certains éléments d'une langue. "Définitions " peu cochées par les enseignant (e)s des groupes, lusitanophone et arabophones, dont il a été question ci-dessus.

C'est que cette conception n'est guère courante dans l'enseignement / apprentissage des L2, pas plus qu'elle ne l'est dans la formation initiale ou continue des enseignants de français L2. La règle grammaticale est raisonnée, dans cette conception, à la manière dont deux philosophes européens ont, il y a bientôt un siècle, raisonné la connaissance scientifique : Gaston Bachelard dans son Nouvel esprit scientifique [1934] et Karl R. Popper dans Logik der Forschung («Logique de la découverte scientifique») [aussi de 1934]. Ils s'inscrivaient dans des traditions philosophiques différentes, plutôt française pour Bachelard, plutôt allemande pour Popper. Mais l'un et l'autre affirment que la théorie est inévitablement antérieure à l'observation scientifique, qu'il n'existe pas de science sans $a$ priori conceptuels ou philosophiques. Toute connaissance scientifique du monde physique serait une réponse à des questions d'ordre plus ou moins métaphysique. Ce que Thomas $\mathrm{S}$. Kuhn résumera dans son ouvrage The Structure of Scientific Revolutions [1962 et 1970] en disant qu'il n'existe aucune possibilité d'expliciter le sens d'une expression comme «ce qui se trouve réellement dans la nature" en dehors d'une théorie particulière. Conception qui s'oppose donc, assez radicalement, à la conception descriptiviste telle que nous l'avons précisée.

Pourquoi si peu d'enseignants de français L2, natifs ou non natifs, formés en France ou hors de France, cochent-ils nos deux « définitions » constructivistes? Sans doute, parce que la majorité des grammairiens ont fait durant des siècles, et font encore, comme si leurs inévitables a priori métaphysiques n'étaient pas des hypothèses, mais seraient inhérents à «l'esprit humain ", et partant relèveraient d'une «logique » universelle et quasi a-historique. Pourquoi questionner des a priori ainsi naturalisés? De nombreux 
linguistes ont certes critiqué, depuis plus d'un siècle, les catégories (en particulier les sept à dix catégories de mots ou "parties du discours ») que les grammairiens admettent souvent comme allant de soi, mais leurs critiques et propositions novatrices n'ont que très marginalement modifié la coutumière fabrique des règles grammaticales (voir sur ce point, Jean-Pierre Lagarde 1988). En outre, parmi les rares enseignants de français L2 qui cochent l'une ou l'autre de nos « définitions » constructivistes, il en est qui pensent que le savoir des grammairiens et des linguistes est non seulement relatif (hypothèse ou dont on suppose y invitent) mais aussi que, au moins dans les débuts de l'enseignement / apprentissage d'une L2, mieux vaut beaucoup d'usage et peu de règles que l'inverse conviction qui était déjà celle de Quintilien ( $1^{\text {er }}$ siècle de notre ère).

Une remarque pour conclure cette partie. Nous avons présenté nos trois (pré)conceptions de ce qu'est une règle grammaticale comme si elles s'étaient succédé historiquement, à la manière des «révolutions scientifiques" telles que les conçoit Kuhn. C'est que, pour expliciter ce qu'on entendait par là, nous avons fait appel aux théorisations d'ordre philosophique qui en ont été faites, et que ces théorisations, portant sur les progrès ou "révolutions» des mathématiques et des sciences de la nature, s'échelonnent historiquement. Il en va, nous semble-t-il, différemment dans « les sciences humaines et sociales ", et particulièrement en science grammaticale ou linguistique.

41 Son histoire atteste que, des siècles avant Comte, Bachelard ou Popper, certains grammairiens étaient déjà, à leur façon, descriptivistes ou même constructivistes. Et de nos jours, ces trois conceptions co-existent, sinon dans l'esprit des grammairiens ou des linguistes contemporains, du moins dans l'esprit de nombre d'enseignants actuels du français L2. Quant aux qualificatifs que nous avons donnés à ces trois (pré)conceptions, ils sont loin d'être partagés par tous. Un seul exemple. On trouve, dans la $8^{\text {ème }}$ édition de la "grammaire française" de M. Grevisse (1964), ce paragraphe que nous citons intégralement (y compris ses gras et italiques) :

La grammaire descriptive expose l'usage linguistique d'un groupement humain à une époque donnée. Elle se borne ordinairement à constater et à enregistrer le “ bon usage", c'est-à-dire l'usage constant des personnes qui ont souci de bien parler et de bien écrire. C'est alors la grammaire normative ou, selon la définition habituelle, "l'art de parler et d'écrire correctement".

Ce qui revient à mêler nos (pré)conceptions juridique et descriptiviste. Il n'en demeure pas moins qu'il n'existe pas actuellement, alors qu'on dispose des moyens informatiques pour travailler sur d'immenses corpus, une seule «grammaire française » qui ait œuvré sur l'ensemble des usages actuels, tant oraux qu'écrits, du français. Toutes, y compris celles qui se prétendent les plus descriptives, ont travaillé sur un certain «bon usage » plus ou moins libéralement interprété.

\section{De l'art d'enseigner les règles grammaticales d'une L2}

On distingue couramment deux manières d'enseigner les règles grammaticales d'une L2. Soit l'on procède déductivement, de la règle élaborée par les grammairiens à des exemples qui l'appliquent, lesquels peuvent être suivis de quelques autres exemples qui la limitent (les « exceptions » censées « confirmer la règle »). Soit l'on procède inductivement, allant d'exemples de la L2 choisis à cet effet à la règle qui explicite, en termes métalinguistiques, ce que ces exemples ont de grammaticalement commun. 
Il est tentant d'associer la démarche déductive à la conception juridique telle que nous venons de la préciser, et la démarche inductive à notre conception descriptiviste. Le droit n'est-il pas traditionnellement enseigné en partant des lois telles que formulées techniquement par les légistes ou les jurisconsultes, avant de voir comment elles ont été interprétées et appliquées par la jurisprudence. Inversement, les sciences de la nature, et parfois même les mathématiques, ne sont-elles pas enseignées à partir de diverses expériences permettant aux élèves d'appréhender concrètement ce à quoi peut renvoyer, dans un réel qui leur est sensiblement observable, la loi ou la règle abstraite que le maître leur enseigne ensuite. Mais si démarche déductive-conception juridique opposée à démarche inductive-conception descriptiviste aide à mieux comprendre ce qui oppose l'enseignement grammatical déductif (de la règle aux exemples) à l'enseignement grammatical inductif (des exemples à la règle), ces deux associations ne tiennent pas compte, ou pas assez, de ce qui peut se passer effectivement dans les classes où l'on enseigne / apprend une L2.

\section{De l'enseignement déductif des règles grammaticales d'une L2}

De nos jours, la règle est enseignée aux débutants, pour peu que le maître la pratique, dans leur L1 ${ }^{21}$, avant de l'être, peu à peu et à mesure qu'ils progressent dans la L2, dans la langue qu'ils sont en train d'apprendre. Une fois ainsi transmise et dûment expliquée en L1, la règle est mise en œuvre dans divers exercices à même d'aider et de vérifier que les élèves la mettent correctement en pratique. Ce qu'on appelle les «thèmes grammaticaux » en relèvent, qui consistent à «traduire ${ }^{22}$ des exemples de la L1 en L2, en appliquant la règle qui leur a été préalablement inculquée.

Cet enseignement déductif de règles grammaticales portant sur une L2 en s'appuyant sur la L1 des apprenants a été âprement débattu, en particulier au XVIII ${ }^{e}$ siècle. S'il s'est maintenu en France durant les deux siècles suivants, c'est pour des raisons d'ordre plus institutionnel que didactique : en procédant à la manière des enseignants des «langues mortes» (latin et grec), les enseignants des «langues vivantes » (anglais, allemand, espagnol L2) se voulaient professionnellement leurs égaux, et donc mieux à même d'exiger d'être traités comme eux. Mais hors de France, au cours du xix siècle, on fit de plus en plus appel à des natifs du français pour l'enseigner en tant que L2, natifs qui ignoraient souvent presque tout de la L1 de leurs élèves, laquelle par ailleurs n'était pas toujours dotée de règles par les grammairiens ${ }^{23}$. Il en résulta que ces enseignants, ne pouvant suivre la démarche déductive, l'ont délaissée ${ }^{24}$. Elle n'en perdure pas moins ça et là, pour peu qu'on y dispose de maîtres plus ou moins bilingues L1-L2, que les autorités administratives y privilégient la « transmission » d'un savoir savant sur les L2 enseignées, ou que ces maîtres bilingues y trouvent un moyen de conforter leur autorité25.

\section{De l'enseignement inductif des règles grammaticales d'une $L 2$}

Cette démarche inductive est, depuis environ deux siècles, la plus pratiquée, mais elle l'a été bien auparavant et de façon plus diverse que la démarche déductive. Telle que nous l'entendons, il y a enseignement inductif de la règle lorsque les élèves ont été quelque peu familiarisés aux données sur lesquelles elle porte avant qu'elle ne soit explicitée soit par le maître, soit par les élèves fortement guidés et aidés par le maître. Mais cet enseignement inductif a été aussi pratiqué sans que la règle soit explicitée : la pratique 
intensive d'exemples l'appliquant est alors supposée l'inculquer sans qu'on ait, d'une manière ou d'une autre, à l'expliciter ${ }^{26}$. On peut ainsi distinguer trois façons de pratiquer l'enseignement inductif d'une règle grammaticale relative à une L2: explicitée par le maître ; plus ou moins explicitée par les élèves ; non explicitée.

Si le maitre se propose d'expliciter lui-même la règle, il commence par familiariser ses élèves aux données que cette règle est censée régir, soit en s'appuyant sur un texte où ces données sont plus ou moins récurrentes, soit sur une série d'exemples qui ne font pas texte mais qui sont censés l'illustrer clairement, des « exemples parlant » comme on dit parfois.

S'il part d'un texte, il attire l'attention des élèves sur les données qu'il juge pertinentes pour la règle, en constitue un petit corpus dont il explique ensuite les régularités au moyen de la règle qui lui a permis de le constituer. Procédé circulaire, typique de la démarche inductive.

S'il part d'une série d'exemples, il n'en va guère différemment, les exemples étant choisis à partir de la règle qu'il veut enseigner. Ainsi préparés (manipulés ?), les élèves sont alors mieux à même de comprendre la règle qui a ordonné ces exemples, règle que le maître donne en général dans la L1 de ses élèves plus que dans la L2 qu'ils sont en train d'apprendre, et qu'il formule plus ou moins telle qu'elle l'a été par les grammairiens, en usant de leurs termes techniques si les élèves les connaissent déjà, en les reformulant en mots plus courants s'ils ne les connaissent pas. Il en vérifie ensuite la compréhension par ses élèves, en leur demandant d'en faire l'application dans divers exercices ("à trous ", mots isolés à mettre en phrase selon la règle, etc.), puis en la rappelant chaque fois que les élèves l'oublient dans des contextes plus naturels, moins scolairement contraints.

51 L'article Grammaire du Dictionnaire de Buisson (voir ci-dessus à propos de la conception descriptiviste) cite un inspecteur général qui caractérise ainsi cette manière de procéder (1911 : 747, nos italiques) :

Cette méthode [...] part d'exemples, d'où les règles se dégagent d'elles-mêmes; elle appelle l'attention des élèves sur plusieurs phrases où les mêmes faits de langage se reproduisent sous des formes différentes, et les acheminent à trouver d'eux-mêmes la conclusion qui s'impose. Voyant la grammaire se faire sous leurs yeux, contribuant à la faire, les élèves s'y intéressent comme à leur œuvre propre.

Ce qui peut être vrai. Mais pourquoi avoir mis les quatre verbes soulignés à la voix ${ }^{27}$ pronominale, considérée le plus souvent comme une variante de la voix active (quand le sujet «fait l'action») ? Telle qu'elle est employée ici, cette voix pronominale n'a ni le sens réfléchi (il se regarde dans la glace), ni le sens réciproque (ils se regardent). Les règles se dégagent d'elles-mêmes signifie qu'elles dévoilent ce qu'elles sont sans qu'aucun "sujet " extérieur ne les dévoilent. Dire que les mêmes faits de langage se reproduisent sous des formes différentes, c'est dire que ces «faits" ne sont pas observables dans ces formes, qu'ils relèvent de quelque abstraction dont on ne dit pas qui l'a opérée. Et dire que la conclusion du raisonnement inductif s'impose, sous-entendu d'elle-même, c'est dire que ce raisonnement ne doit rien à celui (celle) qui raisonne. Bref, ces emplois pronominaux suggèrent que les règles grammaticales ne résultent pas du travail des grammairiens ou des linguistes, qu'elles (pré)existeraient dans la «nature» des langues, et que la "méthode" inductive préconisée permettrait à des élèves qui les ignorent de les découvrir par eux-mêmes. Il y a là un réalisme des règles grammaticales quelque peu naïf, qui n'est guère éloigné de ce que F. Brunot entendait par les «lois scientifiques de la linguistique historique et comparée» (voir aussi ci-dessus notre conception 
descriptiviste), lesquelles ne seraient que "des faits observés et généralisés ${ }^{28}$. Mais les règles grammaticales d'une langue "ne se sont pas faites" d'elles-mêmes, et les découvrir quand on ignore tout de la manière dont elles ont été originellement raisonnées n'est pas toujours facile. Et si les élèves y parviennent, leur « découverte » doit en général beaucoup au maître qui les connaît et qui en a choisi les exemples. Toujours la circularité du raisonnement inductif.

Afin que les enseignant(e)s que nous avons en formation prennent conscience de cette difficulté, que minimise ou occulte la conception descriptiviste, nous leur proposons un exercice portant sur l'emploi de pour et parce que dans des phrases "complexes ${ }^{29}$. Certains observent que pour est suivi d'un verbe à "l'infinitif passé », alors que parce que est suivi d'un "verbe conjugué »; d'autres se perdent dans diverses considérations portant sur la «cause » et la "conséquence »; avant qu'un(e), rarement plus dans des groupes de vingt à soixante, ne parvienne, devant notre refus des propositions précédentes, à « découvrir » la règle dissimulée dans les exemples, à savoir que le sujet de la proposition principale et la subordonnée est, ou doit être, le même avec pour, alors qu'il est, ou peut-être, différent avec parce que. Et si l'on demande s'il(ou elle) l'a réellement découverte dans cet exercice, s'il(elle) ne l'a pas redécouverte pour l'avoir déjà apprise (à propos du français ou d'une autre langue), presque toujours l'enseignant(e) avoue qu'il (elle) la connaissait déjà. Les exercices de grammaire inductive de ce type sont, de fait, des exercices de remémoration du savoir grammatical que possèdent déjà les élèves, et s'ils ne disposent du savoir pertinent pour l'exercice, les exemples, si bien choisis soientils, restent muets.

D'où peut-être, devant les difficultés auxquelles se heurtait la « méthode » précédente, la vogue en France, dans les années 1960-70, des "exercices structuraux ", transposition française des patterns drills en vogue aux Etats-Unis dans les décennies précédentes. Mais alors que ces patterns drills étaient souvent précédés d'une explication précisant le point grammatical traité, et suivis d'une Generalization permettant au maître de corriger les généralisations non acceptables que les apprenants pouvaient faire du pattern enseigné, les «exercices structuraux» ou les «tables de structure » n'étaient, le plus souvent, ni précédés d'une explication grammaticale, ni suivis d'une « exploitation » visant à vérifier ce que les apprenants faisaient, dans d'autres contextes (hors exercice), de la « structure » inculquée par la seule répétition intensive d'exemples censés la manifester. Comme si cette pratique intensive et quelque peu mécanique était à même d'imprimer dans le cerveau des apprenants cette " structure », sans que les élèves aient à faire appel à quelque savoir métalinguistique que ce soit.

Le psittacisme de cette pratique était alors présenté et justifié comme une application «scientifique " d'un «structuralisme » différent du «structuralisme " de Saussure, et d'un «behaviorisme » qui refusait de prendre en compte ce qui peut se passer dans la «boîte noire » (black box) de «l'esprit humain ». Deux théories importées des Etats-Unis servant également à justifier l'emploi de ces «exercices structuraux» dans les « laboratoires de langue ». Mais cette apparence de modernité et de scientificité masquait mal la croyance que les « structures » ainsi inculquées, tout comme les « règles » dans la pratique inductive de la conception descriptiviste, seraient inhérentes à la L2, comme si elles en étaient l'ossature ou la charpente "cachée». Ce qui différenciait ces deux pratiques était que les élèves n'étaient plus incités à inférer consciemment d'exemples choisis à cet effet la "règle ", puisque la répétition intensive de ces exemples était 
supposée l'inscrire dans les comportements langagiers des apprenants, sans avoir besoin de recourir à leur « boîte noire».

\section{Pour un enseignement constructiviste des règles grammaticales d'une L2} des années 1970, ce que nous avons appelé des «exercices de conceptualisation». La critique que nous en faisions était à la fois pratique et théorique. Du point de vue pratique, transposés dans certains manuels de français L2 sous forme de «tables de structure ", les patterns drills entraînaient les élèves à surgénéraliser la "structure » sousjacente à ces « tables ». Après avoir répété mécaniquement une série de "substitutions » proposées par une «table » (Je ferai danser les filles / chanter les garçons / écouter des disques / rire les spectateurs...), certains d'entre eux produisaient : *je ferai dire les garçons / *écouter les filles... Phrases grammaticalement possibles selon la «structure», mais inacceptables en français. Une série de "transformations " (je sors la voiture / le chien / les assiettes... => je la (le, les) sors) pouvait aboutir à je sors la nuit => *je la sors, tout aussi inacceptable. Et sur le plan théorique, ces "exercices structuraux ", emblématiques d'une linguistique appliquée alors triomphante, relevaient souvent plus de la théorie grammaticale traditionnelle que de la théorie "structurale », même réputée nord-américaine.

Ce qui singularise fondamentalement nos "exercices de conceptualisation ", c'est la volonté de prendre en compte, dans le « ici et maintenant » de la classe de L2, ce qui peut se passer dans la tête des élèves, dans leur «boîte noire", quand ils cherchent à comprendre, à s'expliquer à eux-mêmes, les difficultés qu'ils ont à intérioriser les régularités grammaticales de la L2. Difficultés dont nous faisons l'hypothèse qu'elles sont certes liées aux différences et ressemblances entre les régularités de leur L1 (ou d'une première L2) et celles de la L2 qu'ils sont en train d'apprendre, mais aussi, et peut-être plus, à ce qu'ils ont antérieurement appris du savoir des grammairiens ou des linguistes quant aux régularités de cette L1 (ou cette première L2). En d'autres termes, à leur « passé grammatical ", aux techniques qu'ils ont déjà apprises pour analyser et raisonner grammaticalement les régularités des langues qu'ils pratiquent déjà. Il s'agit de faire en sorte, en classe et au stade d'apprentissage de la L2 auquel ils sont parvenus, de tester ce qu'ils re-mobilisent de ce savoir savant, certains plus que d'autres, pour résoudre leurs difficultés d'intériorisation des régularités de cette $\mathrm{L} 2$.

En effet, si le maître cherche à aider ses élèves en leur donnant les règles que les grammairiens ou les linguistes ont élaborées quant à la L2 qu'il enseigne, ces règles ne correspondent en général pas à leurs « besoins » d'apprentissage, au stade où ils en sont. Faisant appel à des données de la L2 qu'ils n'ont pas encore pratiquées, elles leur sont difficilement compréhensibles. Est-il possible de vraiment réfléchir sur ce qu'on ne connaît pas encore ? À quoi s'ajoute que ces règles ne sont que rarement contrastives par rapport à leur L1, et qu'elles peuvent recourir à une terminologie trompeuse, les mêmes termes ou des termes voisins renvoyant à des notions grammaticales autres que celles auxquelles ils sont accoutumés.

Un « exercice de conceptualisation » repose sur la conviction, proche de ce que Kuhn dit des théories dans l'étude de la nature, que si un élève se pose une question d'ordre grammatical quant à ses difficultés d'intériorisation de la L2, il a les moyens «théoriques » d'y répondre par lui-même. Ce type d'exercice n'est que l'occasion, pour 
cet élève, d'avancer en classe, devant ses pairs et devant son maître, la réponse qu'il apporte plus ou moins spontanément à sa question, réponse dont il ne sait pas quelle est la validité ou l'utilité par rapport à ce qu'il a déjà appris de la L2. C'est une façon de la tester au sein du groupe-classe. Validée par celui-ci, elle restera provisoire ou transitoire, l'apprentissage ultérieur de la L2 pouvant conduire à la modifier ou à l'abandonner. Sorte d'adjuvant cognitif, bricolé en classe, à l'intériorisation préalable, routinière ou communicative, de certaines régularités de la L2, un « exercice de conceptualisation » ne peut être présent dans un manuel de L2, sauf à titre d'exemple possible.

Ainsi conçu, un « exercice de conceptualisation » ressemble à un exercice de grammaire inductive explicitée (voir ci-dessus), mais il en diffère profondément, au moins sur les quatre points suivants :

a. Il n'est programmable ni dans un manuel de L2 destiné à des publics de L1 différentes et de traditions grammaticales diverses (manuels « universels »), ni guère plus dans les manuels (" contextualisés ») destinés à des publics de même L1, et ayant appris, à l'école, à peu près la même représentation grammaticale de cette L1. Il est une pédagogie des erreurs commises par certains élèves et non par d'autres. Par exemple, dans une classe où des élèves ayant l'allemand pour L1 apprennent le français L2, certains disent Paul a sa moto et d'autres Paul $a{ }^{*}$ son moto. Divergence dans l'intériorisation des adjectifs possessifs du français qui peut être l'occasion de ce type d'exercice.

b. On y part non d'exemples choisis par le maître (ou le manuel) pour que les élèves en induisent la règle qui a permis de les choisir (exercice de grammaire inductive explicitée), mais d'un petit corpus de phrases produites par les élèves eux-mêmes, qu'elles soient grammaticalement acceptables ou non en L2. Au maître de dire celles qui le sont et celles qui ne le sont pas, en ayant la précaution, pour certaines, de demander aux élèves qui les sont produites dans quel contexte ils les imaginent, car ce qui est agrammatical dans un contexte peut être plus ou moins acceptable dans un autre. On part donc d'un petit corpus de phrases correspondant à la question posée (dans l'exemple ci-dessus : Comment «fonctionne » en français son et $s a$ ?), phrases attestant de l'intériorisation réussie ou non par le groupe-classe d'un micro-domaine grammatical de la L2. Un petit corpus à même de donner au groupeclasse l'occasion de réfléchir grammaticalement, en ayant recours à ce qu'ils ont retenu du savoir des grammairiens qui leur a été antérieurement enseigné, sur les emplois corrects ou non de son et sa en français.

c. Réflexion qui peut conduire, dans ce même exemple, un élève, celui qui s'est posé la question à l'origine de l'exercice ou un autre, à se dire «j'ai compris comment ça fonctionne ». Et à montrer à ses pairs et à son maître, par exemple au moyen de simples gestes si l'usage ouvert de la L1 est proscrit ou s'il n'est pas possible, l'idée qu'il se fait de ce « fonctionnement. Un geste montre qu'en français sa est lié au nom qui le suit (moto); un autre que son équivalent en allemand est lié à un mot qui le précède, Paul. C'est une "conceptualisation», parmi d'autres possibles, qui répond à la question que sa et son posaient au groupe-classe. C'est une sorte de «règle» dont il est clair qu'elle n'est ni une invention ni une découverte de l'élève qui la propose, ces deux gestes pouvant suffire à la rappeler à l'ensemble de la classe, y compris à ceux qui ne l'ont pas suivie. "Règle » contrastive, et donc plus complexe que celles dont peuvent user les natifs du français L2 (du type si l'on dit la moto, alors c'est sa moto), mais qui convient mieux aux difficultés que des élèves germanophones ont à s'approprier l'emploi de son et sa en français.

d. Cette «règle » est ensuite testée sur les autres phrases du petit corpus qu'ont produit les élèves, puis sur de nouvelles phrases produites également par eux afin d'en éprouver la généralité. Mais ce n'est pas au maitre d'avancer des contre-exemples faisant appel à des données qu'ils n'ont pas encore apprises. Le groupe-classe peut ainsi se constituer un cahier de grammaire qui lui est propre, comprenant les petits corpus de phrases qu'il a produits à 
propos de ses difficultés d'intériorisation des régularités de la L2, ainsi que les « règles » qu'il a bricolées pour les résoudre.

61 Pour mener à bien ce type d'exercice, il faut que le maître soit à même de suivre des cheminements grammaticaux autres que ceux auxquels il est lui-même accoutumé, qu'il ne soit pas persuadé que les meilleures règles pour ces élèves sont celles qu'il a dans la tête, et qu'il admette, par simple expérience ou par réflexion épistémologique, une conception des règles grammaticales plus constructiviste que descriptiviste. Si ce n'est pas le cas, l'exercice de conceptualisation se transforme aisément en exercice de grammaire inductive explicitée.

Une dernière remarque sur notre conception constructiviste du savoir grammatical. Ce que nous qualifions ainsi est, en épistémologie des sciences, relativement récent, et nombre de grammairiens ou de linguistes continuent à se réclamer de la conception descriptiviste. C'est dans le Cours de linguistique générale de Ferdinand de Saussure (cours recueilli par deux de ses élèves et publié en 1916) que nous en avons repéré, pour la première fois en linguistique, la présence (1973:23) :

D'autres sciences opèrent sur des objets donnés d'avance et qu'on peut considérer ensuite de différents points de vue; dans notre domaine, rien de semblable. [...] Bien loin que l'objet précède le point de vue, on dirait que c'est le point de vue qui crée l'objet, et d'ailleurs rien ne nous dit d'avance que l'une de ces manières de considérer le fait soit antérieure ou supérieure aux autres.

Ce passage a été souvent interprété en termes kantiens (relatifs aux a priori de la connaissance), mais il est aussi interprétable selon ce que Bachelard ou Popper diront un peu plus tard du savoir scientifique dans les sciences de la nature, même si, pour ces deux épistémologues, les objets des sciences de la nature ne sont pas, contrairement à ce que dit le Cours, "donnés d'avance ", mais eux aussi « construits ", inscrits dans une théorie préalable, pour être étudiés scientifiquement.

Toutefois, ce que nous appelons la (pré)conception constructiviste semble plus ancienne. Nous avons cité un inspecteur général qui, dans le Dictionnaire de F. Buisson, disait que les élèves «voyant la grammaire se faire sous leurs yeux, contribuant à la faire [...], s'y intéressent comme à leur œuvre propre ». Un siècle et demi auparavant, on lit sous la plume de Claude Lizarde de Radonvilliers, conscient «qu'un grand nombre de personnes " ont de la peine «à se persuader que la Grammaire soit inutile pour apprendre les Langues» (1768: 224), leur objecte que les élèves qui suivent sa " méthode de la double version ${ }^{30}$ ", dont le savoir des grammairiens est quasi absent, se feront par eux-mêmes «une grammaire personnelle, qui aura pour eux une véritable utilité, parce qu'elle sera le fruit de leurs réflexions, \& non pas celles d'autrui ».

Deux énoncés quasi paraphrastiques, qui ne se différencient que par les (pré)conceptions grammaticales qu'ils engagent. Notre inspecteur général ne doute pas que les élèves parviendront par eux-mêmes, à partir d'exemples choisis à cet effet, à « la conclusion qui s'impose ", à la règle ou au principe impliqué dans les exemples, dont il est persuadé que c'est un « fait de langage ». Radonvilliers raisonne tout autrement. Il reconnaît que déjà, en son temps, on « est assez porté à croire que les Règles de la Grammaire sont les principes de la Langue », mais c'est, à ses yeux, " une erreur : il n'y a d'autres principes dans les Langues que l'usage » (ibid.: 228). Il ajoute toutefois (ibid., nous soulignons) que « les élèves, à mesure qu'ils feront des progrès, découvriront eux-mêmes des principes », et non les principes de la langue qu'ils apprennent. Car, pour lui, «telle est la nature de l'esprit humain » que « dès qu'il a acquis plusieurs idées, il les combine, il les compare, il 
en observe les rapports » (ibid.: 229), ce qui aboutirait selon lui, quasi inévitablement, à cette " grammaire personnelle ».

Ce qui différencie la conception constructiviste telle que nous l'entendons de celle de Radonvilliers, c'est que, à nos yeux, "l'esprit humain", au moins celui des élèves qui commencent à apprendre une L2, ne parvient à raisonner grammaticalement sur cette langue, que s'il a été préalablement armé,, à propos d'une L1 ou d'une première L2 apprises scolairement, de quelques rudiments conceptuels empruntés à l'une ou l'autre des théories qu'ont pu inventer les grammairiens ou les linguistes.

\section{Conclusion, ou de la « culture grammaticale » du français}

«Ramenons, écrivait Radonvilliers, la Grammaire à son véritable usage : c'est la science des gens habiles qui veulent connoître une Langue à fond » (ibid. : 227). Il est clair que, de nos jours, ce n'est pas le but de la très grande majorité de ceux qui apprennent une L2. Il ne s'agit pas d'en faire des grammairiens ou des apprentis linguistes.

Mais certaines langues plus que d'autres exigent, à des stades un peu avancés de leur apprentissage, un enseignement d'une autre représentation grammaticale que celle que les apprenants ont pu se constituer dans leurs successives « conceptualisations ». Il s'agit de la représentation grammaticale, qualifiée souvent de "traditionnelle», qui a été transmise par des siècles de culture, scolaire ou non, d'une langue, à tel point qu'elle a fini par être intégrée dans ses usages ordinaires, tant écrits qu'oraux. C'est particulièrement le cas du français, dont la "grammaire traditionnelle» est plus utile, dans les usages quotidiens de cette langue, que nombre de ses représentations grammaticales supposées plus savantes. Ne serait-ce que pour en consulter les grammaires et dictionnaires courants, ou pour utiliser sur ordinateur ses logiciels "correcteurs", autant d'usuels fondés, à quelques innovations près, sur cette représentation traditionnelle.

Un indice de cette acculturation différenciée selon les langues est les réponses que des enseignants de français L2 apportent spontanément à la question suivante ${ }^{31}$ : Quand vous pensez au français, quelle est la première règle grammaticale qui vous vient à l'esprit ? Environ les deux tiers d'entre eux, quelle que soit leur origine et quel que soit le pays où ils exercent leur métier, citent en premier la règle des participes passés avec avoir, et en second celle qu'ils formulent généralement ainsi: «quand deux verbes se suivent, le second est à l'infinitif ». Deux règles qui n'ont d'utilité que dans l'écrit normé, pour bien orthographier les verbes dont l'infinitif est en er et le participe passé en é(e), suffixes strictement homophones à l'oral mais que l'orthographe néanmoins distingue. Si l'on pose aux enseignants de français L2 non natifs de cette langue la même question, mais à propos de leur L1 ou d'une autre L2 qu'ils maîtrisent déjà, les premières règles qui leur viennent à l'esprit, quand il leur en vient une (l'anglais étant souvent pratiqué actuellement sans guère se référer aux règles de grammaire), sont à la fois plus diversifiées et beaucoup moins tributaires de l'orthographe de ces langues.

Pourquoi les francophones quelque peu lettrés accordent-ils la priorité à ces deux règles ? Nombre d'entre eux, fussent-ils des universitaires, ne suivent oralement la première que quand ils y pensent, quand ils se rappellent qu'ils sont censés l'appliquer aussi aux verbes en $i r$ ou re, là où la différence entre verbe et participe s'entend à l'oral ${ }^{32}$. Et seuls les 
francophones soucieux d'orthographe ou attentifs à leur logiciel de correction suivent la seconde, parce qu'elle échappe oralement à toute sanction sociale au moins pour les verbes en $e^{33}$. Trois correcteurs du journal Le Monde attestent que ces deux règles, les plus emblématiques de la grammaire «traditionnelle » du français, sont aussi celles qui sont les moins suivies par les journalistes francophones actuels (voir Rousseau M., Houdart, O., Herlin, R., 2016). Et si l'on pose à des enseignants du français L2 non natifs de cette langue, la même question à propos de leur L1 ou d'une autre L2 qu'ils maîtrisent déjà, les premières règles qui leur viennent à l'esprit, quand il leur en vient une (l'anglais étant souvent pratiqué sans guère se référer à cet "art des lettres»), sont à la fois plus diversifiées et beaucoup moins tributaires de l'orthographe de ces langues.

Pourquoi, en français, cette priorité accordée à deux règles qui sont, spontanément, si peu suivies? Notons d'abord qu'elles sont conformes à l'étymologie du grec grammatiké, d'où procède grammaire et ses équivalents dans les autres langues européennes, qui est d'abord l'art de lire et d'écrire les lettres (grammata). Et qu'elles permettent de distinguer ceux qui ont appris à distinguer un "participe passé » d'un «infinitif» de ceux qui ne l'ont pas appris ou qui l'ont oublié, ce qui n'est pas sans conséquence dans la manière dont on peut être socialement perçu, au moins parmi ceux qui, pour reprendre M. Grevisse, " ont souci de bien parler et de bien écrire ». Elles impliquent une norme, au sens «ce qui doit être" et non au sens «ce qui est conforme à la majorité des cas", norme socialement évaluative à laquelle la millénaire culture du français a été, nous semble-t-il, plus attentive que la culture d'autres langues européennes.

\section{BIBLIOGRAPHIE}

BESSE, Henri (1995). « Ferdinand Brunot, méthodologue de l'enseignement de la grammaire du français ", Histoire, Épistémologie, Langage, tome XVII, fasc. 1, 41-74. Saint-Denis (France) : Presses Universitaires de Vincennes.

BESSE, Henri (2001). « Peut-on naturaliser l'enseignement des langues en général et celui du français en particulier ", Le français dans le monde - Recherches et Applications, Théories linguistiques et enseignement du français aux non-francophones (S. Bouquet, coord.), juillet, 29-58. Paris : CLE International.

BESSE, Henri ; Rémy PORQUIER (1984). Grammaires et didactique des langues. Paris : Crédif-Hatier (collection « Langues et apprentissage des langues »).

BRUNOT, Ferdinand [1919) [1909]. L'enseignement de la langue française. Ce qu'il est - Ce qu'il devrait être dans l'enseignement primaire. Cours de méthodologie recueilli par N. Bony. Paris : A. Colin. BUISSON, Ferdinand, sous la direction de (1911). Nouveau dictionnaire de pédagogie et d'instruction primaire. Paris : Hachette.

LAGARDE, Jean-Pierre (1988). « Les parties du discours dans la linguistique moderne et contemporaine ", Langages, $n^{\circ}$ 92, 93-108. 
RADONVILLIERS, Claude François Lizarde de (1768). De la manière d'apprendre les langues. Paris :

Saillant.

ROUSSEAU, Martine ; HOUDART, Olivier ; HERLIN, Richard (2016). Retour sur l'accord du participe passé et autres bizarreries de la langue française. Paris : Flammarion.

SAUSSURE, Ferdinand (1973) [1916]. Cours de linguistique générale. Édition critique préparée par

Tullio de Mauro. Paris : Payot.

\section{NOTES}

1. Contrairement à certains tenants des méthodes directe, audio-visuelle ou communicative qui pensent qu'une L2 peut être acquise comme on a acquis sa L1.

2. La difficulté à enseigner une langue à ce qu'on appelle «les enfants-loups", quand on les découvre passés cet âge, le suggère.

3. Expression relativement répandue chez les enseignants de français L1, mais qui ne semble pas être antérieure à la fin du XVIII ${ }^{\mathrm{e}}$ siècle. Dans Émile ou de l'éducation, c'est en un sens voisin que Jean-Jacques Rousseau emploie le mot grammaire: "Les enfants ont, pour ainsi dire, une grammaire de leur âge, dont la syntaxe a des règles plus générales que les nôtres, et si l'on y faisait attention, l'on serait étonné de l'exactitude avec laquelle ils suivent certaines analogies, très vicieuses si l'on veut mais régulières et qui ne sont choquantes que par leur dureté ou parce que l'usage ne les admet pas.». Par exemple, il arrive qu'un enfant francophone, habitué à dire dormir-dormira et sortir-sortira, dise mourir- ${ }^{*}$ mourira. Notons que Rousseau modalise (voir son pour ainsi dire) l'emploi qu'il fait ici de grammaire, ce qui suggère que cet emploi est alors peu courant, est une sorte de néologisme de sens.

4. La phonétique, l'intonation, la gestualité, le lexique, la pragmatique ne relèvent pas, ou peu, de la grammaire prise en ce sens, même si des grammairiens et des linguistes ont pu en tenir compte. 5. Il suffit de comparer les programmes scolaires du primaire quant au nombre d'heures consacrées à l'enseignement de la "grammaire » et de l'orthographe de la L1 pour se faire une idée de la plus ou moins grande importance qu'on accorde à cette culture.

6. C'est ainsi que l'ont traduite en japonais des enseignants de français L2.

7. Distinction qui n'est pas récente, même si on la formule de nos jours autrement. Un grand grammairien français du XVIII ${ }^{\mathrm{e}}$ siècle, Nicolas Beauzée, estimait ainsi que seule «la Grammaire générale " était une science, "parce qu'elle n'a pour objet que la spéculation raisonnée des principes immuables \& généraux du Langage», que "ses principes ne supposent que la possibilité des langues ", et qu'ils sont donc antérieurs aux langues observables, alors qu' « une Grammaire particulière est un art ", en ce qu'elle ne peut être " que le résultat des observations faites sur les usages préexistants ».

8. Certains migrants peuvent vivre des années durant dans leur pays d'accueil sans parvenir à en pratiquer la langue comme ses natifs la pratiquent, gardant leur «accent " et commettant nombre analogies (morpho-syntaxiques ou lexicales) qui ne sont pas dans l'usage de ces natifs. Eux aussi apprennent à la fois moins et plus que ce que leur environnement leur « enseigne ».

9. La notion de « grammaire intériorisée » est proche de celles de « compétence linguistique » ou " d'intuition linguistique », mais ces notions sont liées à un " locuteur-auditeur idéal », figure du linguiste qui étudie une langue plus que de ses locuteurs réels, qu'ils soient natifs ou non de cette langue. Il existe certes des enseignants de L2 que ne parlent pas ou qui parlent mal la L2 qu'ils enseignent, tout comme il existe des natifs de cette langue qui l'enseignent en ignorant presque tout de ce que les grammairiens ou les linguistes ont pu en dire, mais ces cas sont relativement marginaux. 
10. Avant la Renaissance (fin $\mathrm{XV}^{\mathrm{e}}$ - début $\mathrm{XVI}^{\mathrm{e}}$ siècles), seules les langues « savantes " (grec, latin, hébreu) étaient dotées de règles grammaticales, et elles étaient enseignées le plus souvent à partir de ces règles; les langues "vulgaires ", celles parlées par le vulgus ou le tout-venant, en étaient jusqu'alors dépourvues, et étaient en conséquence apprises par le seul usage, à la manière dont un enfant apprend sa L1.

11. Voir à ce propos la première partie de l'ouvrage Grammaires et didactique des langues que j'ai publié (Paris: Didier 1984) avec Rémy Porquier. J'y montre comment deux phénomènes grammaticaux du français, particulièrement difficiles à qui apprend à cette langue en tant que L2, sont diversement représentés par les grammairiens et linguistes, celui de la morphologie et des « temps » du « verbe » en français, et celui des « articles » du français.

12. Le bon usage de Maurice Grevisse en est un bon échantillon.

13. Il y a plus d'un siècle, le philosophe américain Charles Sanders Peirce (1839-1914) disait (nous le traduisons) : «Nous sommes si loin d'avoir le droit de conclure qu'une théorie est la vérité même, que nous ne pouvons pas même comprendre ce que cela veut dire. » Propos que corrobore une spécialiste actuelle de la philosophe des sciences physiques, Soazig Le Bihan : « Les lois de la physique, a fortiori celle de la biologie, ne sont que des hypothèses probables, non des vérités d'expérience. [...] Dès lors, la question de la vérité d'une théorie perd son sens, et la problématique de l'invention remplace celle de la découverte. »

14. Par exemple, là où la tradition grammaticale occidentale, d'origine gréco-latine, distingue de sept à dix catégories grammaticales (les "parties du discours» ou "espèces de mots »), la tradition grammaticale arabe en distingue trois, une nominale (où se retrouvent nos "noms", « adjectifs », « pronoms »...), une verbale (où se retrouvent nos «verbes » et une partie de leurs «temps»), et une qui assure les liens entre les deux premières (où se retrouvent plus ou moins nos prépositions ", « conjonctions » et autres « mots de liaisons »).

15. Les cultures traditionnelles chinoise et japonaise se sont passées de tout savoir grammatical durant des siècles, lequel n'a commencé à s'y introduire que lorsque les missionnaires occidentaux ont cherché à décrire grammaticalement les langues chinoises et japonaise.

16. Dans les années 1960-1990, c'était le cas de nombreux élèves britanniques venant de l'enseignement public, y compris des grammar schools, les programmes scolaires ne comprenant plus de cours de grammaire.

17. Dans la version que nous utilisons actuellement, laquelle est le résultat de divers remaniements de ses versions antérieures.

18. Selon le Petit Robert.

19. Celui qui, au bureau du greffe, transcrit et conserve les actes originaux des procès.

20. Auteur d'un Cours de philosophie positive en six volumes paru entre1830 et 1842 et traduit, entre autres langues, en anglais en 1856.

21. Pour ce qui est du latin, les règles ont longtemps été enseignées directement en latin, mais le latin était, au moins pour ceux qui l'étudiaient jusqu'au XIX ${ }^{\mathrm{e}}$ siècle, davantage une langue seconde qu'une langue étrangère (les élèves y étaient plus ou moins accoutumés par les prières et les offices religieux).

22. Rappelons que traduire, au sens habituel du mot, requiert un certain bilinguisme que n'ont pas ceux qui commencent à apprendre une L2.

23. Entre autres, nombre de langues africaines.

24. Triomphent alors les méthodes qui préconisent d'enseigner "directement» en L2, de la méthode Berlitz à la méthode audio-visuelle, en passant par la méthode directe.

25. Que l'on pense à ces classes de L2 ou à ces cours universitaires de L2 où nombre d'apprenants sont des natifs de la L2 qui y est enseignée, et qui partant disposent d'une intuition grammaticale (d'une " grammaire intériorisée ») supérieure à celle de leur maître.

26. C'est plus ou moins ainsi que procède Nebrija dans le Libro quinto de sa Gramática de la lingua castellana que hizo el Maestro Antonio de Lebrija [1492]. Ce « cinquième livre » est destiné à los que de 
estraña lengua querran deprender les règles grammaticales du castillan, et Nebrija les enseigne alors au moyen de paradigmes d'exemples les illustrant sans guère les expliciter.

27. Formes du verbe exprimant le rôle du sujet dans l'action signifiée par le verbe.

28. Pour qui s'intéresserait plus avant à l'enseignement de la grammaire selon F. Brunot, nous renvoyons à Besse (1995).

29. Emprunté à un manuel de français $\mathrm{L} 2$, cet exercice comprend cinq exemples avec pour et parce que (Pour avoir recueilli quelques menus objets lors des inondations [...], deux personnes ont été condamnées [...] par le tribunal de Grande instance vs. Parce qu'ils avaient recueilli quelques menus objets lors des inondations [...], le tribunal de Grande instance a condamné deux personnes [...])

30. On y familiarise l'élève à la pratique de la L2, en partant de textes extraits d'auteurs ayant illustré cette langue, textes qui lui sont présentés d'abord traduits littéralement dans sa L1 («version des mots»), ensuite traduits plus élégamment («version de la pensée »). Pour Radonvilliers (1768: 225) : « La lecture est une espèce de conversation, \& doit produire les mêmes effets."

31. Question que nous avons posée, au cours de notre carrière de formateur d'enseignants de français L2, à des centaines de natifs et non natifs de cette langue.

32. Ainsi peut-on entendre ou lire, la fresque que ce peintre a *peint dans sa jeunesse, au lieu de a peinte, ou la faute qu'ils ont * commis, en place de commise.

33. Rien ne différencie à l'oral les terminaisons de j'ai mangé et je vais manger; ce qui n'est pas le cas pour j'ai cueilli et je vais cueillir, j'ai vu et je vais voir, j'ai mis et je vais mettre.

\section{RÉSUMÉS}

Sont d'abord quelque peu précisés les termes de l'intitulé de cet atelier, en particulier pourquoi une barre entre enseignement et apprentissage, pourquoi des guillemets à "grammaire ", et pourquoi n'y est-il question que du français. Trois points sont ensuite abordés. La polysémie actuelle du mot grammaire, ou de ses équivalents dans les autres langues européennes, que l'on peut réduire à trois sens fondamentaux ("grammaire intériorisée », "représentations grammaticales » et "théories grammaticales»). Les conceptions les plus courantes que l'on se fait de ce qu'est une règle grammaticale : une l'assimilant à la loi juridique ; une autre, à une « loi de la nature »; une troisième, à un artefact du grammairien. Trois conceptions auxquelles correspondent plus ou moins trois manières de faire apprendre cette règle, l'une déductive, l'autre inductive, et la troisième d'ordre constructiviste. Ces quatre parties sont suivies d'une brève conclusion portant sur la « culture grammaticale » du français.

The author first clarified the terms used in this workshop, particularly his reasons for putting a slash between teaching and learning, writing grammar between brackets and talking about French only. He then discusses three points. The current polysemy of the word "grammar", or its equivalents in other European languages, which can be brought down to three basic senses ("interiorized grammar", "grammatical representations", and "grammatical theories"). The most widespread notions of what a grammatical rule is i.e. first assimilation to judicial law; second, to "natural law"; third, to a grammarian's artefact. Three notions implying more or less three manners of teaching that rule i.e., first, an deductive one; second, an inductive one; the third one being of constructivist order. A brief conclusion bearing on the "grammatical culture" of French follows those four parts. 
INDEX

Mots-clés : enseignement des langues, grammaire, règle de grammaire, Français Langue Étrangère (FLE)

Keywords : language teaching, grammar, grammatical rule, French as a Foreign Language (FFL)

\section{AUTEUR}

HENRI BESSE

ENS de Lyon 